\title{
Diacronie
}

Studi di Storia Contemporanea

$N^{\circ} 21,1 \mid 2015$

Le città di Babele

\section{Le locande della solidarietà (Genova, XVIII secolo)}

\section{Cinzia Bonato}

\section{(Q) OpenEdition \\ Journals}

\section{Edizione digitale}

URL: http://journals.openedition.org/diacronie/1857

DOI: 10.4000/diacronie.1857

ISSN: 2038-0925

\section{Editore}

Association culturelle Diacronie

\section{Notizia bibliografica digitale}

Cinzia Bonato, «Le locande della solidarietà (Genova, XVIII secolo) », Diacronie [Online], N² 21, 1 | 2015, documento 3, Messo online il 01 mars 2015, consultato il 19 avril 2019. URL : http://

journals.openedition.org/diacronie/1857 ; DOI : 10.4000/diacronie.1857 


\title{
Diacronie
}

3/

\section{Le locande della solidarietà (Genova, XVIII}

\section{secolo)}

\author{
Cinzia BONATO *
}

La storiografia ha finora guardato alle locande come pericolosi luoghi di pernottamento e ritrovo per migranti, vagabondi, devianti o come alberghi per accogliere turisti, visitatori o commercianti. In realtà, esse erano anche luoghi di aggregazione di persone e nuclei familiari problematici sia genovesi sia provenienti da altri stati, in cui la convivenza permetteva di tessere legami ed elaborare strategie di solidarietà che si estendevano e collegavano anche al loro esterno. In esse si operava la più evidente saldatura tra la città notturna, operosa e fervente di attività illegali o dormiente e bisognosa di un'alcova, e diurna, legale e produttiva. Attraverso l'analisi di tutte le informazioni relative alle locande e ai suoi avventori contenute nelle Cause Criminali dell'ospedale di Genova, Pammatone, potremo addentrarci in alcune strutture ricettive genovesi e scoprire pratiche e strategie di sopravvivenza messe in atto dalla popolazione per eludere la necessità di rivolgersi agli istituti assistenziali.

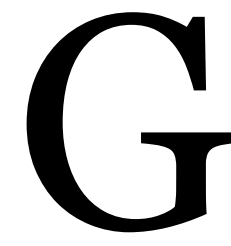

li studi sul pauperismo si sono finora preoccupati di catalogarne le caratteristiche, disegnando un quadro della povertà che ne sottolineasse i confini decisamente inclusivi e l'ineluttabilità di cadervi per la maggior parte della popolazione, a partire dalle categorie più a rischio ${ }^{1}$. D’altro

\footnotetext{
${ }^{1}$ La bibliografia è vasta; cito qui HUFTON, Olwen H., The Poor of Eighteenth-Century France, 1750-1789, Oxford, Clarendon Press, 1974; PULLAN, Brian, Poveri, mendicanti e vagabondi (secoli XIV-XVII), in Storia d'Italia. Dal feudalesimo al capitalismo, Torino, Einaudi, 1978, pp. 51-83, che richiama le considerazioni fatte da GUTTON, Jean Paul, La società e i poveri, Milano, Mondadori, 1977; WOOLF, Stuart J., Porca miseria. Poveri e assistenza nell'età moderna, Roma-Bari, Laterza, 1978, che dedica all'argomento il primo capitolo, "I poveri e la
} 
canto, quelli sull'assistenza, concentrati sui grandi reclusori costruiti nel XV secolo proprio in risposta alla crescente e dilagante miseria provocata da epidemie e carestie, si sono soffermati sul controllo sociale esercitato dagli istituti assistenziali, sostenendo o meno la loro capacità di esercitarla o la possibilità di eluderla da parte della popolazione. Tra i primi, troviamo il filone inaugurato da Michel Foucault, che ha evidenziato la capacità delle istituzioni di esercitare un controllo sociale pressoché totale e arbitrario sulla popolazione ${ }^{2}$. Tra i secondi, invece, si sono posti in prima linea gli studiosi di genere, che fin dagli anni '8o del Novecento hanno compiuto ricerche per propugnare la capacità di reazione degli assistiti e il loro ruolo attivo.

In effetti, in età moderna sfuggire alla povertà e all'assistenza poteva essere un'impresa ardua, ma è proprio il terreno di queste difficoltà che bisogna dissodare se si vogliono comprendere le risorse che la società stessa era in grado di attivare. Infatti, attraverso un'analisi più approfondita delle strutture ricettive cittadine emergono i metodi e le strategie che le persone elaborarono per riuscire a sfuggire a quella morsa o per attivare reti di solidarietà che permettessero di eludere il ricorso all'assistenza, o di limitarlo a un soccorso esterno (distribuzione di minestra, di pane o di denaro). Evidenziare queste strategie può servire per capire che cosa, nel tessuto cittadino, si fosse effettivamente attivato a sostegno di parte dei suoi abitanti, soprattutto di quelli più sfortunati; se la povertà era pressoché ineluttabile, non esisteva soltanto il ricorso all'assistenza per combatterla, soprattutto nelle grandi città.

Con l'intento di sondare questi spazi, vorrei qui proporre l'analisi di alcuni documenti che permettono di addentrarsi in determinate strutture ricettive genovesi e di scoprire pratiche e strategie di sopravvivenza messe in atto dalla popolazione per sfuggire al controllo esercitato dagli istituti assistenziali. Lo farò focalizzando l'attenzione su alcuni tipi di locande genovesi della seconda metà del Settecento, quelle che offrivano rifugio per la notte a persone che vivevano in condizioni di marginalità o di estrema povertà. In esse gli avventori potevano fermarsi per un giorno, per qualche settimana o anche, in alcuni casi, per mesi, a volte anni; in caso di prolungata frequentazione, avevano l'opportunità di formare reti di solidarietà a supporto di

società nell'Europa occidentale”, pp. 3-46; LOMBARDI, Daniela, Povertà maschile, povertà femminile. L'ospedale dei Mendicanti nella Firenze dei Medici, Bologna, Il Mulino, 1988; ZAMAGNI, Vera (a cura di), Povertà e inovazioni istituzionali in Italia. Dal Medioevo a oggi, Bologna, il Mulino, 2000.

2 Per una teoria del controllo sociale che vuol dimostrare la passività della popolazione davanti alle istituzioni: FOUCAULT, Michel, Storia della follia nell'età classica, Milano, Rizzoli, 1963 e ID., Sorvegliare e punire. Nascita della prigione, Torino, Einaudi, 1976. Diversi studiosi si occuparono di dimostrarne invece il ruolo attivo: cfr. per esempio il volume curato da FERRANTE, Lucia, PALAZZI, Maura, POMATA, Gianna (a cura di), Ragnatele di rapporti. Patronage e reti di relazione nella storia delle donne, Torino, Rosenberg \& Sellier, 1988. 
situazioni di disagio o di necessità, tramite le quali era possibile garantire la sopravvivenza e quindi evitare di ricorrere alla rete assistenziale3.

Se, dunque, la storiografia ha finora guardato alle locande come pericolosi luoghi di stazionamento e di ritrovo per migranti o vagabondi provenienti dalle campagne, o per devianti ${ }^{4}$, e come alberghi per accogliere turisti e visitatori o, nel caso di città portuali come Genova, persone la cui attività è ricollegabile a quella del porto5, qui inizieremo a parlarne presentandole come luoghi di aggregazione di persone e nuclei familiari problematici, sia genovesi sia migranti, in cui la convivenza permetteva di tessere legami ed elaborare strategie di solidarietà che si estendevano e collegavano anche al loro esterno.

In esse si operava la più evidente saldatura tra la città notturna, operosa e foriera di attività illegali o dormiente e bisognosa di un'alcova, e diurna, produttiva, nella quale ogni azione doveva esser ripagata; tra i gestori, che intendevano attirare un certo tipo di clientela, e gli avventori dei loro locali.

\section{I documenti dell'Arte degli osti e locandieri}

Prima di utilizzare i documenti che hanno ispirato quest'articolo, ho compiuto un sopralluogo nell'Archivio di Stato della città di Genova sperando di trovare ulteriore documentazione e di poter così ampliare la ricerca. Purtroppo, ho scoperto che $\mathrm{i}$ documenti che testimoniano dell'attività dell'Arte degli osti e locandieri sono davvero pochi. L'unico fascicolo, prezioso tanto quanto danneggiato, copre quasi una trentina d'anni: si apre nel 1748 e si estende fino al $1774^{6}$. In questo periodo furono rilasciate cinquanta autorizzazioni: una media di due ogni anno, dovute, probabilmente, al naturale turnover delle attività, poiché la città attesta, nel periodo, una decrescita demografica e perciò risulta difficile ipotizzare un accresciuto bisogno di strutture ${ }^{7}$. Le cifre richieste per le aperture regolari variavano dalle 100 alle 200 lire, la cui corresponsione, in alcuni casi (purtroppo non è possibile capire in quali) poteva anche

\footnotetext{
${ }^{3}$ A Genova, essa era costituita dall'Albergo dei Poveri, il polivalente reclusorio cittadino; dai due ospedali, Pammatone e gli Incurabili; dal sistema di distribuzione del pane nei vari quartieri.

${ }^{4}$ TULIANI, Maurizio, Osti, avventori, e malandrini: alberghi, locande e taverne a Siena nel suo contado tra Trecento e Quattrocento, Siena, Protagon Editori Toscani, 1994; FABBRI, Pier Giovanni, Artigiani, botteghe, osterie e locande: ricerche sui luoghi del lavoro a Cesena nei secoli $X V$-XIX, Cesena, Società di studi romagnoli, 2001.

${ }^{5}$ DAVİ, Mauro, La città ospitale. Locande e alberghi a Genova dal '6oo a oggi, Genova, Sagep, 1988.

${ }^{6}$ Archivio di Stato di Genova, Arti 177, fascicolo 14.

${ }^{7}$ GRENDI, Edoardo, in Introduzione alla storia moderna della Repubblica di Genova, Genova, Bozzi, 1976, p. 59, ci informa sul calo demografico della città, che scende dagli 82.395 abitanti del 1743 agli 81.624 del 1773.
} 
avvenire a rate. Il dato completo dovrebbe però includere le molte attività irregolari, aperte e condotte illegalmente: nel fascicolo appaiono solo i casi più evidenti, che sono appuntati in piccoli fogli dove appaiono brevi annotazioni sui trasgressori. Possiamo inoltre ipotizzare che alcune locande furono aperte per manifestare apertamente il loro carattere solidale e protettivo: tra le autorizzazioni ne sono registrate cinque (il 10\% del totale) rilasciate a stranieri, ossia a tre irlandesi, un francese e un milanese, che probabilmente si caratterizzarono proprio per la provenienza estera dei loro gestori e perciò, in gran parte, dei loro avventori.

Ma i documenti attraverso i quali si può iniziare ad affrontare il problema tramite un'analisi qualitativa non riguardano la corporazione, bensì provengono dalle testimonianze rese al Magistrato di Pammatone, l'ospedale maggiore di Genova, in processi riguardanti furti e parti illegittimi ${ }^{8}$. L'ospedale aveva una sua magistratura che si occupava di tutti i casi riguardanti i dipendenti o avvenuti al suo interno. Tra le quattro filze di Cause Criminali da essa prodotte, assumono una grande rilevanza le testimonianze rese in seguito alla riattivazione, nel 1759, di una legge emanata nel lontano 1481, e che fino a fine secolo riscosse un notevole successo. Essa permetteva di incriminare i padri naturali dei figli illegittimi abbandonati alla ruota di Pammatone e di obbligarli così a pagare il mantenimento del bambino tramite la sola deposizione di prima istanza della partoriente o della puerpera. Tra le loro numerose testimonianze, si trovano molte dichiarazioni e diversi racconti che riguardano le più note locande genovesi del Settecento.

\section{Vivere in locanda, costruire reti di solidarietà}

Analizzando i documenti ospedalieri possiamo prima di tutto scoprire alcune caratteristiche degli avventori delle locande genovesi. Iniziamo dalla professione e dai tempi di permanenza: possiamo trovare alcune informazioni nella testimonianza resa dalla locandiera Pellegrina Folle fu Paolo nel 1773 che, interpellata come testimone in un processo per furto, descrisse la sua attività dicendo:

Vengono a dormire nella mia locanda povera gente, e fra questi più di frequente vi vengono un certo Francesco Borsotto di professione stoppiere, ed un certo Baldassarre di professione camallo da carbone, e vi vengono puranco qualche donne e di ordinario una detta la Mora, che si nomina Massina, e qualche altra

\footnotetext{
${ }^{8}$ Sull'ospedale: CARPANETO DA LANGASCO, Cassiano, Pammatone. Cinque secoli di vita ospedaliera, Genova, Ospedali Civili, 1953.
} 
volta una soprannominata la Perucchiera, ed altra di sua compagnia che vanno dimandando elemosina 9 .

Non erano dunque solo i mendicanti a soggiornare per pochi soldi nella sua locanda, anzi: la maggior parte delle persone erano povere, ma praticavano una professione. Nella sua lista, troviamo infatti stoppieri, camalli, parrucchiere e poi la Mora, "che passa per meretrice" ${ }^{10}$. Certo, il confine tra guadagnare grazie al proprio lavoro e dover mendicare era labile: molti mestieri, tra i quali anche molti corporati, erano in crisi, e in tempi di magra non era così insolito affiancare la questua a una professione, o praticare più lavori ${ }^{11}$.

Una tale situazione di precarietà poteva rendere più conveniente dormire in locanda, piuttosto che affittare un mezzano e sobbarcarsi il costo dell'affitto. Così, gli avventori potevano soggiornarvi per diverso tempo: c'era chi vi si fermava solo una notte, altri la sceglievano come soluzione temporanea e vi stavano per qualche giorno, ma vi era chi decideva di starvi qualche mese o era obbligato a farlo dalle circostanze, come Antonio Cevasco di Giuseppe, genovese, che nel 1794 disse di esser stato costretto ad andare alla locanda perché

\begin{abstract}
mia moglie dieci mesi circa [or] sono, essendosi partita di casa mia per alterchi avuti con me, ed avendo portato seco il mattarazzo, lenzuoli ed altro di casa, io essendo rimasto più mesi solo, senza saper nuova della medesima, ed avendo venduto il saccone che mi era rimasto ed il letto mi trovai in necessità massime nella circostanza del freddo di andare a dormire nella locanda, come feci in quella di Ponticello ${ }^{12}$.
\end{abstract}

Antonio non aveva dunque molta scelta: era senza mobili, senza biancheria, senza un tetto sulla testa nonché senza moglie: per sua fortuna, la locanda poteva fornirgli

\footnotetext{
${ }^{9}$ Archivio degli Ospedali di Genova (d'ora in poi AOG), Cause (Criminalium). Dall'anno 1772 al 1787 (d'ora in poi CC I), n. 24, B.IV-6 (filza) A. Per furto di un lenzuolo commesso da un'ammalata del presente Ospedale, 18 ottobre 1773.

${ }^{10}$ Ibidem, testimonianza di Baldassarre Villa.

${ }^{11}$ Per un quadro generale della situazione dei mestieri genovesi nel Settecento: MASSA, Paola, Lineamenti di organizzazione economica in uno stato preindustriale. La Repubblica di Genova, Genova, ECIG, 1995. Per uno studio più delineato: GRENDI, Edoardo, Introduzione alla storia moderna della Repubblica di Genova, Genova, Bozzi, 1976, pp. 85-94. Per quanto riguarda, invece, una descrizione delle persone che dichiararono di svolgere più di un mestiere: BONATO, Cinzia, Molto più che pazienti. L'ospedale di Pammatone e la popolazione della Repubblica di Genova nel XVIII secolo, Tesi di dottorato in Storia moderna, Università degli Studi di Torino, Torino, 19 febbraio 2013; tutor: prof. Luciano Allegra.

${ }_{12}$ AOG, Cause (Criminalium), Dall'anno 1788 al 1802 (d'ora in poi CC III), n. 26, B.IV-8 (filza), Processo c.o Ant.o Caorsi d.o il Bargalino per furto de cuchiaro fatto in spetiaria, 27 maggio 1794.
} 
tutto questo. Nel 1797 Antonio Bevegno fece la stessa cosa e per un periodo ben più lungo. Dieci mesi non erano nulla in confronto ai sei anni durante i quali egli, ogni notte, pagò una locandiera per dormire. Raccontò di essere arrivato da S. Olcese sei anni prima, e di non aver mai avuto «[...] abitazione fissa, solendo vivere alla locanda, fuorché per tre mesi fa, prima di entrare in ospedale, ho preso abitazione in $\mathrm{S}$. Andrea»13. Il suo pellegrinaggio finì, quindi, in corrispondenza con l'impiego finalmente ottenuto in ospedale, che gli poteva garantire un'entrata pressoché sicura con la quale fronteggiare i costi di un mezzano.

La storia di Antonio ci dimostra come le strutture ricettive fossero un punto di riferimento imprescindibile per chi risiedeva nei paesi della Serenissima e decideva di cercare lavoro nella capitale. Egli non era il solo: anche Margherita Rasetto disse di aver dormito sei anni in locanda dopo il suo arrivo da Sori14, mentre le più fortunate sorelle Pedevilla, arrivate da Comago, vi dormirono per quindici giorni, per poi approdare al lavoro di domestiche grazie all'appoggio della donna per la quale la madre era stata balia. Possiamo trarne la conclusione che le locande fossero un buon surrogato per chi arrivava dalla riviera o dall'interno e non aveva una buona rete di conoscenze che potesse facilitare il suo inserimento nella realtà lavorativa genovese: il forte turnover degli avventori permetteva di conoscere molta gente, e quindi di costruirsi quella rete. Inoltre, le strutture ricettive garantivano un luogo di stazionamento flessibile e temporaneo, gradito soprattutto quando fornivano ai loro clienti un letto a buon mercato, come faceva Piero, in Portoria, che chiedeva due soldi per notte ${ }^{15}$, o la locandiera che, per pura carità, diede gratuitamente da mangiare e da dormire a Daria Di Casa ${ }^{16}$. Ci si fermava nell'attesa, più o meno lunga, di riuscire ad allacciare rapporti, riattivare conoscenze e trovare un'occupazione, ma molte volte la sistemazione provvisoria diveniva stabile, prolungandosi nel tempo. Possiamo dunque supporre che, nonostante la larga clientela, il ricavo ottenuto dai locandieri non fosse troppo alto, visti i bassi prezzi; per questo possiamo immaginare che il caso di Gio' Batta Calcina, che pur conducendo una locanda era anche tessitore di seta ${ }^{17}$, non fosse per nulla isolato.

\footnotetext{
${ }^{13}$ AOG, CC III, Processo di furto di una camiccia commesso dal servitore Antonio Bevegni a danni dell'Opera Pia, 30 gennaio 1797.

${ }_{15}^{14}$ AOG, CC II, s. i., 14 giugno 1792.

${ }^{15}$ AOG, CC III, s. i., 1 marzo 1782. Vi soggiornava Angela Calcagno, mendicante.

${ }^{16}$ AOG, Cause (Criminalium). Dall'anno 1773 al 1827 (d'ora in poi CC II), n. 25, B.IV-8 (filza), senza intestazione (d'ora in poi s. i.), 6 settembre 1791.

${ }^{17}$ AOG, Cause (Criminalium). Dall'anno 1704 al 1785 (d'ora in poi CC IV), n. 21, B.IV-7 (filza), Contra Francesco Martino, 10 settembre 1720.
} 
I documenti di Pammatone permettono di fare un'ulteriore operazione. Lavorando sugli indirizzi delle attività, è stato possibile individuare le loro linee di sviluppo e ricostruire la topografia delle locande genovesi a buon mercato. Sottolineiamo, infatti, che certamente ci saranno state altre strutture ricettive, ma dedicate a un altro tipo di clienti, sicuramente con maggiori disponibilità economiche o con particolari esigenze legate alle attività finanziarie o del porto. Quelle qui individuate sono le strutture dedicate a povera gente, a lavoratori sempre in bilico tra sopravvivenza e carità o a persone che, un lavoro, speravano ancora di trovarlo. La figura 1 rappresenta la loro localizzazione nella Genova racchiusa entro le vecchie mura.

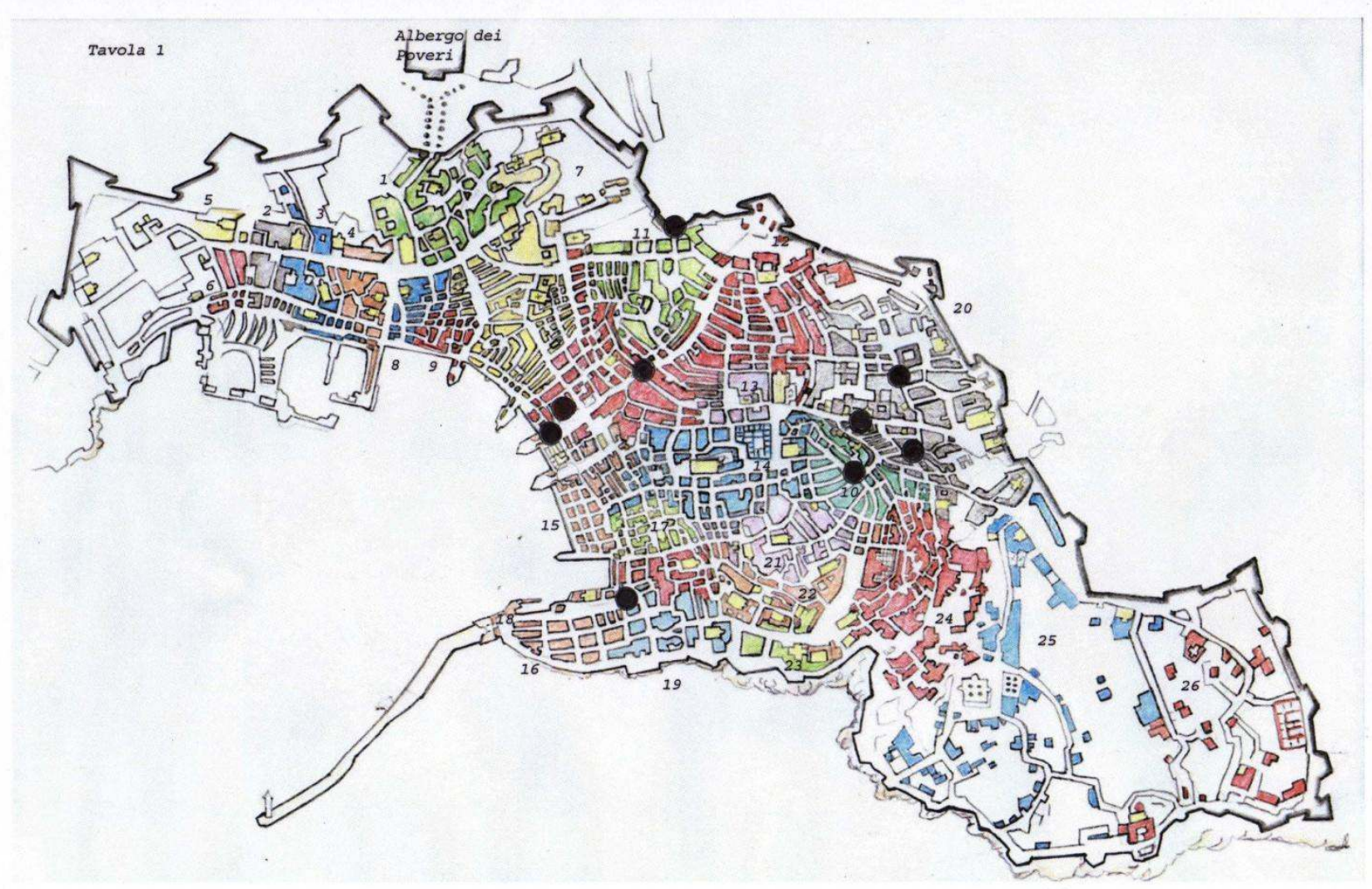

Figura 1. Localizzazione delle locande menzionate nei documenti ${ }^{18}$.

\footnotetext{
${ }^{18}$ La cartina è stata elaborata seguendo le planimetrie disegnate da ACCINELLI, Francesco Maria, in Stato presente della metropolitana di Genova e di tutte le parrocchie tanto in città che nelle Diocesi, Chiese, Conventi, Collegi, Confraternità, Oratorij, ed altre Pie Opere esistenti o annesse al loro territorio: Archivio Storico del Comune di Genova, Fondo Manoscritti.
} 
L’immagine mostra che le locande erano dislocate lungo due direttici. La prima partiva dalla fervente attività del porto e della zona degli affari (il Molo e piazza Reale) e si sviluppava verso l'uscita a nord della città, quella che portava all'entroterra. Era probabilmente questa la traiettoria di chi, dai paesi limitrofi, si recava in città sia per vendere prodotti, che per cercare nuove prospettive di lavoro. E difatti, essa terminava nel cuore degli affari cittadini, ovvero nella zona attorno a piazza Reale. La seconda si concentrava invece a ridosso del quartiere in cui era sorto l'ospedale (S. Stefano e Portoria): una rilevazione che risente, senza dubbio, del tipo di documenti che stiamo analizzando, e che tuttavia dimostra il legame tra questi luoghi di ritrovo per diseredati e l'assistenza, in questo caso ospedaliera. Stupisce solo in parte l'assenza di riferimenti nella zona dell'Albergo dei Poveri, che non fa altro che sottolineare quanto i due istituti (il reclusorio e l'ospedale) avessero funzioni del tutto diverse, in alcuni casi complementari ${ }^{19}$ : all'Albergo i poveri si avvicinavano per ottenere vitto e alloggio, mentre al nosocomio ricorrevano in caso di problemi sanitari, o nella speranza di ottenere un posto di lavoro, come riuscì a fare Antonio; il turnover, nella zona attorno a esso, era certamente più intenso.

Passiamo ora all'analisi quantitativa di alcuni dati riguardanti le trentotto persone di cui è rimasta qualche traccia ospitate in queste locande tra il 1773 e il 1797, ma soprattutto negli anni '9o (ventisei, il 68\%). L'età degli avventori è molto varia: si va dai sedici ai settant'anni, per una media di trentatré anni; non vi era, dunque, una fascia d'età preponderante. È invece evidente la netta prevalenza della presenza femminile: tra gli avventori vi sono infatti nove uomini e ventinove donne. La maggior parte di esse (sedici) era nubile, sei erano vedove, cinque coniugate ${ }^{20}$, mentre la media della loro età è di 29 anni.

Certamente questo dato è influenzato dal tipo di fonte: basta infatti constatare che diciassette donne testimoniarono a Pammatone in quanto madri di un illegittimo ${ }^{21}$. Tuttavia, la loro prevalenza rispecchia il contesto in cui molte di loro erano immerse. Durante il Settecento i mutamenti nel mercato del lavoro, e soprattutto la costante crescita della servitù femminile a discapito della tradizionale servitù maschile ${ }^{22}$, aveva

\footnotetext{
${ }^{19}$ Per esempio, gli orfani erano accolti nell'Albergo mentre gli illegittimi erano esposti nella ruota di Pammatone e qui allevati.

${ }^{20}$ Di due non conosciamo il dato, mentre degli uomini sappiamo che due erano celibi e altrettanti coniugati.

${ }^{21} \mathrm{Vi}$ sono poi due ree di furto e sette testimoni, mentre le rimanenti sono citate da persone coinvolte a vario titolo.

${ }^{22}$ ARRU, Angiolina, Uomini e donne nel mercato del lavoro servile, in GROPPI, Angela (a cura di), Il lavoro delle donne, Roma-Bari, Laterza, 1996, pp. 247-268. La studiosa dimostra che la servitù, nel corso del XVIII secolo, subì un processo di femminilizzazione che portò donne $\mathrm{e}$ uomini a porsi come concorrenti nel mercato del lavoro.
} 
portato molte donne ad allontanarsi dalla famiglia per lavorare in luoghi lontani da quello di origine. Si accentuò dunque la mobilità e l'indipendenza femminile, ma anche la fragilità della loro posizione nella società: una volta giunte nel posto di lavoro, che spesso coincideva con la capitale, esse rimanevano prive di quella rete familiare che garantiva solidarietà e controllo. Era dunque frequente che fossero le donne a candidarsi per un intervento assistenziale, a conferma di una tendenza già rilevata dalla storiografia ${ }^{23}$.

Di fronte a questa condizione, le locande si potevano tramutare in risorsa alla quale attingere per evitare l'assistenza mettendo in pratica strategie individuali. Oltre a offrire un tetto per la notte, esse potevano fornire alle più fortunate un lavoro come serve $^{24}$, o costituire una base per condurre attività illegali come la rivendita di biancheria rubata in ospedale, o la prostituzione ${ }^{25}$. Ad alcune donne il soggiorno poteva essere pagato totalmente $\mathrm{o}$ in parte da un amante ${ }^{26}$, mentre altre, arrivate da lontano, potevano fermarsi per la notte per riporre un bimbo nella ruota ${ }^{27}$.

Ovviamente, per trarre vantaggio dal soggiorno in questi locali occorreva tessere delle relazioni e costruire reti la cui prima area era, il più delle volte, costituita dai gestori e dal personale di servizio. Lo dimostrano due casi. Nel primo, Geronima Parodi, arrivata in città da Bolzaneto e qui fermatasi a lavorare presso un locandiere, partorì a casa del datore di lavoro, aiutata dalle due colleghe (e se possiamo malignamente supporre che il locandiere fosse il padre naturale, ci soffermeremo almeno sull'aiuto fornito, disinteressatamente, dalle altre due serve) ${ }^{28}$; nel secondo, Maria Turcone, convinta dalle false promesse di un uomo a spostarsi da Milano a Genova, dovette sistemarsi in una locanda e riuscì a farlo talmente bene che, quando si ricoverò per una malattia, fu accudita proprio dalle gestrici del locale 29.

La seconda area era formata, invece, dagli altri avventori, coi quali dopo un lungo soggiorno si stringevano amicizie e rapporti utili alla sopravvivenza. E difatti Maddalena Corradina, che si era stabilita nel locale sito a Ponticello da otto anni, ossia

\footnotetext{
${ }^{23}$ L'intervento assistenziale era rivolto principalmente alle donne: cfr. LOMBARDI, Daniela, Povertà maschile, povertà femminile cit.

${ }^{24}$ AOG, CC II, s. i., 4 ottobre 1792; AOG, CC II, s. i., 8 aprile 1793.

${ }^{25}$ Per il caso di rivendita di biancheria: AOG, CC I, A Per furto di un lenzuolo commesso da un'ammalata del presente Ospedale, 18 ottobre 1773. Per quello in cui la donna si prostituiva fuori dalla locanda, ma facendo capo a essa: AOG, CC III, s. i., 11 dicembre 1791.

${ }^{26}$ AOG, CC II, s. i., 11 luglio 1790; AOG, CC II, s. i., 25 marzo 1794; AOG, CC II, s. i., 28 maggio 1794.

${ }^{27}$ AOG, CC II, A. Contro Maria Vassalla di Rapallo per avere posto un suo nipote nel curlo, 2 ottobre 1777.

${ }^{28}$ AOG, CC II, s. i., 20 ottobre 1796.

${ }^{29}$ AOG, CC I, Deposizione di Maria figlia del quondam Michele Turcone milanese del borgo di Porto Comasna, 27 maggio 1785.
} 
da quando si era di fatto separata dal marito, nella sua testimonianza parlò apertamente delle amicizie e conoscenze che lì aveva stretto, mentre Anna, sua madre, disse di vendere «[...] roba che mi portano, e la vendo ossia la pongo fuori dalla porta della locanda ove dormo alla vista di tutti»30. Anna, dunque, aveva ormai una così stretta familiarità col luogo da averne fatto anche il riferimento per il suo lavoro. Ma è Rosa Perazzo, di Sestri Levante, a renderci la più significativa testimonianza dell'importanza di questi legami. Ella depose nel 1792 dicendo:

\begin{abstract}
Venuta in Genova per guadagnarmi il mantenimento mi posi a lavorare nel varego da seta del Signor Gennaro nel caroggio del Fico, ed ove guadagnavo soldi 14 al giorno, ed ho continuato a lavorare sino ad essere venuta in questo spedale. Dormivo nella locanda di Ponticello detta di Baccino, dove lasciavo mio figlio.
\end{abstract}

Rosa affidava dunque il figlio a qualcuno del locale in Ponticello, quello maggiormente nominato nelle testimonianze, per potersi procurare di che sopravvivere: i membri della rete che aveva intessuto le garantivano sufficiente copertura per mantenere i legami familiari31.

Tra gli altri avventori si poteva dunque trovare appoggio, ma anche importanti mediazioni. Un buon esempio è quello di Geronima Rocca, che nel 1791 raccontò che

\begin{abstract}
Sulli primi giorni di gennaio prossimo passato venni in questo spedale ammalata per farmi curare dalla febbre terzana, e vi stiedi da circa due mesi, ed uscita andiedi a dormire nella locanda di Baccino in Ponticello. Vi ritrovai una donna ch'essa pure vi dormiva, e che si chiamava Annetta, e colla quale discorrendo mi disse che mi avrebbe accordata a servire in casa di un certo S.re Azalino, che abitava nella torre di S. Andrea, e di fatto mi vi accordò ${ }^{32}$.
\end{abstract}

In effetti, chi operava come mediatore sapeva di poter trovare, in questo tipo di locande, una buona riserva di mano d'opera: disoccupati, migranti, persone il cui impiego dava a malapena di che sopravvivere, gente, insomma, immediatamente disponibile a essere assunta per qualsiasi mansione - $o$, in alcuni casi, a essere attirata,

\footnotetext{
${ }^{30}$ AOG, CC III, Processo contro Antonio Caorsi detto il Bargalino per furto de cuchiaro fatto in spetiaria, 27 maggio 1794.

${ }^{31}$ AOG, CC III, Deposizione di Rosa Perazza vedova del quondam Cristoforo sul di lei parto illegittimo di una figlia contro Giambatta de Filippi quondam Niccolò, 27 settembre 1792. Purtroppo non sappiamo chi l'aiutasse tenendole il figlio, anche se possiamo supporre che fosse un'altra ospite della locanda.

${ }^{32}$ AOG, CC III, Deposizione di Geronima Rocca, e vedova in seconde nozze di Paolo Sturla, sul di lei parto illegittimo di una figlia contro Giacomo Azzalino, 27 dicembre 1791.
} 
con la scusa, in loschi affari. Ma l'informazione poteva riguardare altro: Se Geronima trovò in locanda chi la sistemò a servizio, Catterina Colucci ricevette invece da una fruttivendola che portava la sua merce nel locale il suggerimento di andare a partorire in ospedale ${ }^{33}$. Sono due esempi di preziose mediazioni, che permettevano alle donne, ancor più se sole, di stringere relazioni utili alla sopravvivenza e di risolvere problemi alquanto urgenti.

Vi era infine chi aveva la fortuna di poter attivare la terza area della rete, quella composta da conoscenze che si avevano in città e che si potevano utilizzare per passare a una condizione di minor precarietà. Lo fecero le sorelle Pedevilla, figlie di Andrea, quando si trasferirono da Comago a Genova. Nel 1786, Lilla disse:

Mio fratello Gio’ prese moglie ed ogni giorno gridavamo, e così per le varie dispute me ne sono andata unitamente a mia sorella, e sono due anni che siamo a Genova. Quando siamo venute a Genova siamo andate a dormire alla locanda in Ponticello dal locandiere Bacino, e vi siamo dormite giorni 15 circa. Fra questo tempo cercavamo d'accordarsi mia sorella per serva, e di fatto si accordò in casa della S.ra Annetta Sicardi perché mia madre l'avea allevato un figliolo, e così era ad essa cognita 34 .

La sua deposizione sottolinea, quindi, l'importanza dei legami tra le donne che risiedevano in città e quelle che abitavano in campagna, creati in questo caso attraverso il baliatico, ma altre volte facenti capo a rapporti di vicinato, di provenienza dallo stesso paese, di amicizia o di parentela: erano queste le relazioni su cui le immigrate potevano contare per trovare una sistemazione lavorativa nella capitale.

\section{Conclusioni}

Con questa breve ricerca abbiamo dunque provato a comprendere quali risorse la società fosse in grado di attivare per far fronte a situazioni di emergenza senza ricorrere al sistema assistenziale cittadino. Oltre a essere un luogo in cui ripararsi per la notte, le locande erano preziosi punti di riferimento per chi aveva la necessità di fare conoscenze e stringere legami di solidarietà con persone che potessero fornire vicendevolmente aiuto e appoggio. Vi ricorrevano poveri, precari, immigrati di entrambi i sessi, ma soprattutto donne. Quest'ultimo punto andrebbe ulteriormente approfondito:

\footnotetext{
${ }^{33}$ AOG, CC III, s. i., 24 dicembre 1781.

${ }^{34}$ AOG, CC I, Processo contro Franca Ferecia detta la Rapalina, ossia la madre de' Biecchi, 16 febbraio 1786.
} 
l'assistenza era rivolta soprattutto alla fascia più debole della popolazione (donne, bambini, anziani); perciò, le strategie femminili utilizzate per evitare di rivolgersi a essa, sia pure per un certo periodo o in determinati cicli di vita, ci racconterebbero qualcosa di più sulla reattività del tessuto cittadino. Come abbiamo già detto, c'è un motivo per cui nelle strutture ricettive genovesi abbiamo riscontrato un alto numero di donne, ovvero il tipo di fonte utilizzata: per questo la prevalenza femminile andrebbe comprovata con ulteriori ricerche d'archivio.

Comunque sia, per molte persone e per parecchie donne, la locanda costituiva il primo passo verso la costruzione di una rete più o meno estesa, secondo la fortuna e le proprie conoscenze di partenza, che permettesse di sopravvivere in città. Dallo studio si può evincere il tipo di contesto entro il quale le locande permettevano l'ingresso. Esso era costituito da una rete formata prima di tutto dai locandieri e dal personale di servizio, ovvero dalle persone di riferimento per tutti gli avventori; poi, dagli altri ospiti, tra i quali era possibile trovare chi poteva fornire informazioni utili, segnalare un impiego o accudire i bambini in orario di lavoro; infine, la rete si apriva verso l'esterno per inglobare parenti o conoscenti che potessero dare una mano per trovare lavoro, a volte alloggio. Entrare in questo tipo di contesto era utile soprattutto a chi proveniva dal contado con l'intenzione di stabilirsi in città; la traiettoria delle strutture ricettive poste tra il porto e la porta a nord delle mura cittadine testimonia come le attività fossero state dislocate sul territorio proprio per rispondere a questa esigenza.

Le locande, dunque, non erano solamente luogo di stazionamento notturno per visitatori, commercianti, migranti o personalità devianti: alcune di esse erano luoghi di incontro che favorivano la formazione di reti di solidarietà utili agli avventori per evitare il ricorso agli istituti assistenziali o, nei casi più gravi, per limitarsi a sfruttarli dall'esterno. 


\section{* L'autore}

Cinzia Bonato è dottoressa di ricerca in storia moderna. Si interessa di storia sociale, dell'assistenza, del lavoro, di genere. Ha pubblicato diversi articoli ed è in corso di stampa presso l'editore Silvio Zamorani il suo libro sul rapporto tra la popolazione e il sistema assistenziale della Repubblica di Genova.

URL: < http://www.studistorici.com/progett/autori/\#Bonato >

\section{Per citare questo articolo:}

BONATO, Cinzia, «Le locande della solidarietà (Genova, XVIII secolo)», Diacronie. Studi di Storia Contemporanea : Le città di Babele, 29/3/2015,

URL:< http://www.studistorici.com/2015/3/29/bonato_numero_21/ >

\section{Diacronie Studi di Storia Contemporanea $\widehat{\beta}$ www.diacronie.it}

Risorsa digitale indipendente a carattere storiografico. Uscita trimestrale. redazione.diacronie@hotmail.it

Comitato di redazione: Jacopo Bassi - Luca Bufarale - Elisa Grandi - Deborah Paci - Fausto Pietrancosta - Matteo Tomasoni - Luca Zuccolo

Diritti: gli articoli di Diacronie. Studi di Storia Contemporanea sono pubblicati sotto licenza Creative Commons 2.5 . Possono essere riprodotti a patto di non modificarne i contenuti e di non usarli per fini commerciali. La citazione di estratti è comunque sempre autorizzata, nei limiti previsti dalla legge. 